\title{
Cost-effectiveness of lowering blood pressure with a fixed combination of perindopril and indapamide in type 2 diabetes mellitus: an ADVANCE trial-based analysis
}

\author{
Paul P Glasziou, Philip Clarke, Jan Alexander, Mohana Rajmokan, Elaine Beller, Mark Woodward, \\ John Chalmers, Neil Poulter and Anushka Patel
}

$\mathrm{R}$ educing the vascular complications of type 2 diabetes mellitus is a global health priority; worldwide the number of people with diabetes is projected to rise from 171 million in 2000 to 366 million in 2030. ${ }^{1}$ Most people with diabetes will die from or be disabled by macrovascular and microvascular complications that can be reduced by blood pressure (BP)lowering therapy. ${ }^{2}$ Traditional strategies have set BP thresholds and targets for treatment. While effective, these strategies are resource intensive, requiring multiple patient visits, careful monitoring of BP and side effects, and the management of complex drug regimens. Hence, few patients ever achieve recommended BP goals. ${ }^{3-5}$ This strategy also neglects patients with diabetes whose BP is not high enough for the arbitrary label of "hypertension" 6 but is still a major determinant of their risk of vascular disease. $^{7}$

A simpler approach in patients with diabetes may be routine BP-lowering therapy, additional to any current treatment and irrespective of initial BP. This is more inclusive and less resource intensive than the target-setting strategy. In testing this alternative strategy, the Action in Diabetes and Vascular Disease: Preterax and DiamicronMR Controlled Evaluation (ADVANCE) trial recently demonstrated the benefits of a fixed-dose combination of an angiotensinconverting enzyme inhibitor, perindopril, and a diuretic, indapamide, versus placebo in preventing macrovascular and microvascular events in participants with type 2 diabetes, irrespective of their BP at inclusion. ${ }^{8}$ This approach was found to reduce cardiovascular mortality (relative risk reduction, $18 \% ; P<0.03)$ and all-cause mortality (relative risk reduction, $14 \% ; P<0.03) .^{8}$ However, concerns have been raised about the expense if it is widely implemented.

We undertook a prospective cost-effectiveness analysis within the ADVANCE trial ${ }^{8-10}$ and report here on the health-related quality of life, resource utilisation, and cost-effectiveness of treatment with perindopril-indapamide compared with placebo.

\section{ABSTRACT}

Objective: To determine the cost-effectiveness of routine administration, irrespective of blood pressure (BP), of a fixed-dose combination of perindopril and indapamide to patients with type 2 diabetes mellitus.

Design, setting and participants: Prospective cost-effectiveness analysis within the Action in Diabetes and Vascular Disease: Preterax and Diamicron-MR Controlled Evaluation (ADVANCE) trial, an international, multicentre, randomised controlled trial of 11140 participants with type 2 diabetes randomly allocated to receive perindopril plus indapamide (4 mg-1.25 mg/day) or placebo.

Main outcome measures: Health-related quality-of-life measured by the EuroQol-5D, resource utilisation, and cost-effectiveness (cost per death averted at 4.3 years' average follow-up, and estimated cost per life-year gained, by extrapolation).

Results: The mean health-related quality-of-life score of survivors was 0.80 (on a 0-1 scale [death to full health]), with no difference between treatment groups. Active treatment reduced hospital admissions for coronary heart disease and coronary revascularisation by $5 \%$. For the Australian participants, perindopril-indapamide cost A $\$ 1368$ per patient during the trial period, but reduced total hospitalisation costs by $\mathrm{A} \$ 410$ and other medication costs (mainly other BP-lowering drugs) by $\mathrm{A} \$ 332$. The absolute reduction in all-cause mortality for the active treatment group was $1.1 \%$, giving a cost per life saved of $A \$ 49200$. Lifetime extrapolation gave an estimated cost per lifeyear saved of $\mathrm{A} \$ 10040$ (discounted at $5 \%$ per year).

Conclusion: The combination of perindopril and indapamide in patients with type 2 diabetes appears to be cost-effective.

Trial registration: United States National Library of Medicine NCT00145925.

MJA 2010; 193: 320-324

\section{METHODS}

\section{Study design and population description}

The ADVANCE trial was a 20-country, randomised controlled, $2 \times 2$ factorial trial involving 11140 participants with type 2 diabetes. One arm of the study compared routine BP lowering based on a fixed-dose (4 mg-1.25 mg per day) perindopril-indapamide combination or matching placebo on top of whatever other hypertensive treatment was being used, if any. ${ }^{10}$ The study began in June 2001 and patient recruitment ended in March 2003; the BP-lowering treatment arm closed in June 2007. Data on mortality, morbidity, quality of life, and hospitalisation came from the main ADVANCE study. We also performed a substudy of the 978 Australian participants, which provided additional information on outpatient resource usage, including outpatient diagnostic investigations and procedures.

People with type 2 diabetes were eligible for the ADVANCE trial if they had been diagnosed at the age of 30 years or older, were aged 55 years or older on entry to the study, and had established vascular disease or at least one other major risk factor for cardiovascular diseases. ${ }^{8}$ There were no BP criteria for inclusion.

\section{Economic evaluation}

We undertook an incremental cost-effectiveness analysis from the perspective of the health care purchaser. Only direct health service costs were included. The analysis was by "intention-to-treat" for both effects and costs: that is, all events and resources were attributed according to a patient's initial randomisation. Thus, perindopril-inda- 
1 Australian unit costs and their sources for the major cost items

\begin{tabular}{|c|c|c|}
\hline Resource item & Unit cost $2007(A \$)$ & Source \\
\hline Standard single GP visit & 28.30 & MBS \\
\hline Perindopril-indapamide (4 mg-1.25 mg daily), per month & 29.83 & PBS \\
\hline Other drugs & Cost per item & PBS \\
\hline The four most frequently used DRGs & Cost per hospital episode & \\
\hline K60B: Diabetes episode without severe complication & 3556 & $\mathrm{NHCDC}$ \\
\hline B70C: Stroke without other complication & 5810 & NHCDC \\
\hline $\begin{array}{l}\text { F62B: Heart failure and shock without catastrophic } \\
\text { complication }\end{array}$ & 4314 & $\mathrm{NHCDC}$ \\
\hline F66B: Coronary atherosclerosis without complication & 1560 & NHCDC \\
\hline
\end{tabular}

$\mathrm{DRG}=$ diagnosis-related group. $\mathrm{GP}=$ general practitioner. $\mathrm{MBS}=$ Medicare Benefits Schedule

$\mathrm{NHCDC}=$ National Hospital Cost Data Collection. $\mathrm{PBS}=$ Pharmaceutical Benefits Schedule .

pamide in the active group was costed on an as-dispensed basis.

The aim of the cost-effectiveness analysis was to estimate the cost per death averted within the trial, and the cost per life-year gained (estimated by extrapolating for individual survivors beyond the mean follow-up period). There were four major data elements: survival to the end of the study; measurements of quality of life; resource usage; and costs for each of the resources used (unit costs).

\section{Outcomes and survival}

All-cause mortality and cardiovascular mortality from the clinical trial have previously been reported. ${ }^{8}$ We calculated the survival time within the study for each treatment group from survival curves. Life expectancy of survivors beyond the close of the study was based on multistate life tables under the assumption of no continuing benefits from the within-trial treatment. These life tables were constructed from parametric survival models, and estimates were based on information about all ADVANCE participants who were alive 2 years after randomisation, including age, sex, smoking status, duration of diabetes and history of major cardiovascular disease.

\section{Quality of life}

To measure health-related quality of life, we administered the EuroQol-5D (EQ-5D) instrument $^{11}$ to all participants from the 20 participating countries. For comparison, version 2 of the 36-item short-form health questionnaire $(\mathrm{SF}-36 \mathrm{v} 2)^{12}$ was also used with the Australian subgroup of participants. Both questionnaires were administered at baseline, and at 2 years and 4 years after randomisation. A comparison of the baseline data ${ }^{13}$ found that the EQ-5D appeared to be as reliable and valid for measuring utility as the SF-36v2, hence the final analysis used the EQ-5D alone.

\section{Resource usage}

Hospitalisation data for participants from all 20 countries were included in an assessment of resource usage, and costs were based on diagnosis-related-group (DRG) categories using core grouping computer software (casemix expert release 2.3.1 AR [Australian revision]-DRG 5.1; 3M Australia, Sydney, NSW), together with information extracted from the National Hospital Cost Data Collection (NHCDC) in Australia. ${ }^{14}$

Of the 978 Australian participants, 948 (97\%) consented to retrieval of Medicare claims data on medical services they received outside of hospital. Data on long-term (outof-hospital) medications were collected as part of the main study, but information about dispensed medications and dosage were only collected for the Australian subgroup.

\section{Unit costs}

Unit costs of resources were allocated as suggested by the Australian Pharmaceutical Benefits Scheme manual of costs. The principal sources for establishing the unit costs of resources were:

- for hospitalisations - DRG costs; ${ }^{14}$

- for outpatient visits and outpatient diagnostic testing — the Australian Medicare Benefits Schedule; and

- for the costs of medications - the Pharmaceutical Benefits Schedule.

All costs are reported in Australian dollars at 2007 values (Box 1).

Our analysis comprised a descriptive phase and a cost-effectiveness analysis. A general-

\section{Statistical analysis}

ised negative binomial regression was used to compare the numbers of medications, DRG episodes, and out-of-hospital visits and procedures between the two treatment groups (as the variance was greater than the mean). Quality-of-life utility scores from the EQ-5D were also compared at the end of the study. No adjustment was made to $P$ values for multiple comparisons.

Mean values for both cost and outcomes with SDs, mean differences and 95\% confidence intervals were reported, as well as cost-effectiveness ratios. A non-parametric bootstrap process, in which participants were sampled with replacement, was used to estimate uncertainty in the estimated results. We report undiscounted 2007 costs and outcomes, along with amounts expressed in net present values using discount rates of 0 (ie, undiscounted) $3 \%, 5 \%$ and $10 \%$ per year. The effect on our main results of uncertainty surrounding some aspects was examined using sensitivity analyses.

\section{RESULTS}

At baseline, characteristics of the 11140 participants allocated to the two treatment groups were similar. ${ }^{8}$ About a third had a prior history of cardiovascular disease. Participants' mean age was 66 years (SD, 6 years); mean number of years since diagnosis was 8 (SD, 6); $43 \%$ were women; $46 \%$ were from Europe, 37\% from Asia, 9\% from Australia, and 4\% each from Canada and New Zealand. At the first (registration) visit, their mean BP was $145 / 81 \mathrm{mmHg}$. Fortyone per cent had a systolic BP less than $140 \mathrm{mmHg}$ and diastolic BP less than $90 \mathrm{mmHg}$, and 25\% were using no antihypertensive medication.

Mean follow-up was 4.3 years, during which allocated treatment was continued for 20001 patient-years (83\% of the time) in the active treatment group and 20849 patient-years (87\%) in the placebo group.

During the study, 879 participants died: $408(7.3 \%)$ in the active treatment group and $471(8.5 \%)$ in the placebo group (relative risk reduction, $14 \%$ [95\% CI, 2\%$25 \%$ ]; $P=0.025$ ). This overall mortality difference was primarily due to the reduction in cardiovascular deaths (Box 2) with active treatment (relative risk reduction, $18 \%$ [95\% CI, 2\%-32\%]; $P=0.027$ ).

\section{Quality of life}

Based on the EQ-5D, ${ }^{15}$ the mean qualityof-life utility assessment (on a scale from 0 [dead] to 1 [full health]) in survivors was 


\begin{tabular}{|c|c|c|c|}
\hline Outcome & $\begin{array}{l}\text { Perindopril- } \\
\text { indapamide } \\
(n=5569)\end{array}$ & $\begin{array}{l}\text { Placebo } \\
(n=5571)\end{array}$ & $\begin{array}{l}\text { Difference } \\
(95 \% \mathrm{Cl})^{\dagger}\end{array}$ \\
\hline Cardiovascular mortality, no. (\%) & $211(3.8 \%)$ & $257(4.6 \%)$ & $0.8 \%(0.1 \%$ to $1.6 \%)$ \\
\hline \multirow[t]{2}{*}{ All-cause mortality, no. (\%) } & $408(7.3 \%)$ & $471(8.5 \%)$ & $1.1 \%(0.1 \%$ to $2.1 \%)$ \\
\hline & \multicolumn{3}{|c|}{ Mean (SD) cost per patient for Australian participants } \\
\hline Hospital costs ${ }^{\ddagger}$ & $7913(11826)$ & $8323(12957)$ & $-410(-2007$ to 1187$)$ \\
\hline Outpatient visit costs $\S$ & $5317(3583)$ & $5389(3807)$ & $-72(-547$ to 404$)$ \\
\hline Intervention costs & 1368 & 0 & 1368 \\
\hline Other drug therapies ${ }^{\S}$ & $8616(6212)$ & $8948(5678)$ & $-332(-1092$ to 428$)$ \\
\hline Total costs (undiscounted) & $23214(16155)$ & $22660(17002)$ & $555(-730$ to 1580$)$ \\
\hline Total costs (discounted at 3\%) & $21811(15159)$ & $21281(15984)$ & 529 (-1476 to 2535$)$ \\
\hline Total costs (discounted at 5\%) & $21001(14565)$ & $20499(15377)$ & 502 (-1425 to 2431) \\
\hline Total costs (discounted at 10\%) & 19223 (13 284) & 18775 (14 070) & $447(-1314$ to 2209$)$ \\
\hline
\end{tabular}

*Costs are in $2007 \mathrm{~A} \$$ (undiscounted unless stated). Subtotals may not sum due to rounding. $†$ Negative cost differences indicate net cost reduction associated with perindopril-indapamide. $\ddagger n=978$. $\S n=948$.

0.80 , with a slight decline over time (from 0.82 at baseline), but with no statistically significant difference between the active treatment and placebo groups. Therefore, we did not adjust the life-years for quality-of-life differences in the economic analysis.

\section{Hospital costs}

The total in-trial average hospital costs per patient, by treatment group and based on Australian DRGs, for both the Australian substudy and all countries, are presented in Box 3. The overall difference in hospital costs between placebo and active treatment groups (placebo minus active) in all countries combined was just over $\mathrm{A} \$ 1$ million (about $4 \%$ of total hospital costs), with most of this arising from reductions in hospitalisations for cardiovascular episodes. Active treatment reduced hospital admissions for coronary heart disease and coronary revascularisation by $5 \%$. There was a statistically significant reduction in cardiovascular events for participants in the active treatment group, with an average cost reduction of $\mathrm{A} \$ 222$ per patient. However, there were wide variations in hospital resource use by ADVANCE study participants in different regions of the world. ${ }^{16}$ In the Australian subgroup, the costs of hospitalisation were about double the average (Box 3), and the cost difference between treatment groups larger, at $\mathrm{A} \$ 410$ per patient (Box 2). This was somewhat higher than costings based on hospitalisation across all countries, cost per patient shown). additional general practitioner visits for prescribing were included (Box 2). Significantly more additional BP-lowering agents were used by the placebo group, and some other cardiovascular agents were also used more in the placebo group (Box 4). The net difference in cost for drugs other than perindopril-indapamide was $\mathrm{A} \$ 332$ per patient (Box 2 ), representing a further $24 \%$ medication cost offset gained by active treatment.

\section{Outpatient costs}

The 948 Australian participants for whom data were retrieved reported an average of 10.2 visits per year to a GP, 3.4 to a medical specialist, and 17.4 to other outpatient services (including diagnostic testing). Over the duration of the trial, none of the differences in outpatient services costs between the perindopril-indapamide and placebo groups were statistically significant (Box 5).

\section{Cost-effectiveness}

mainly due to higher rates of hospitalisation in Australia.

\section{Medication costs}

At the end of follow-up, treatment adherence was $73 \%$ and $74 \%$ in the active and placebo treatment groups, respectively. Participants assigned to perindopril-indapamide used it for an average of 43.1 months (of 52 months' average follow-up), resulting in a cost of A $\$ 1368$ per patient when three assumed
For Australian participants, the average cost of the study therapy at A $\$ 1368$ per patient was partly offset by lower average costs for other types of health care use: hospital costs were on average A $\$ 410$ lower; outpatient visit costs $\mathrm{A} \$ 72$ lower; and other drug therapies were $\$ 332$ lower (Box 2). The combined difference was $A \$ 814$, which represents $60 \%$ of the instudy cost of perindopril-indapamide. At the trial close, the absolute difference in allcause mortality was $1.1 \%$, and the cost

3 Total in-trial average hospital costs per patient by treatment group, based on Australian diagnosis-related groups (DRGs) at 2007 values*

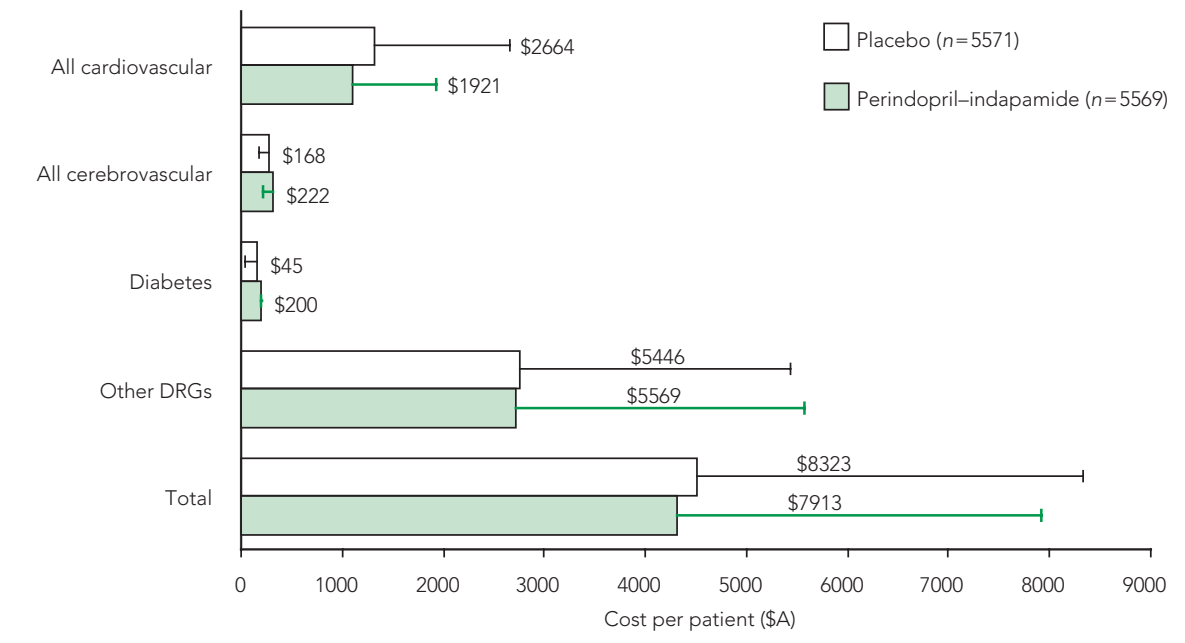

* All 20 countries combined ( $n=11140$; solid bars); and for the Australian participants only $(n=978$; lines, with 


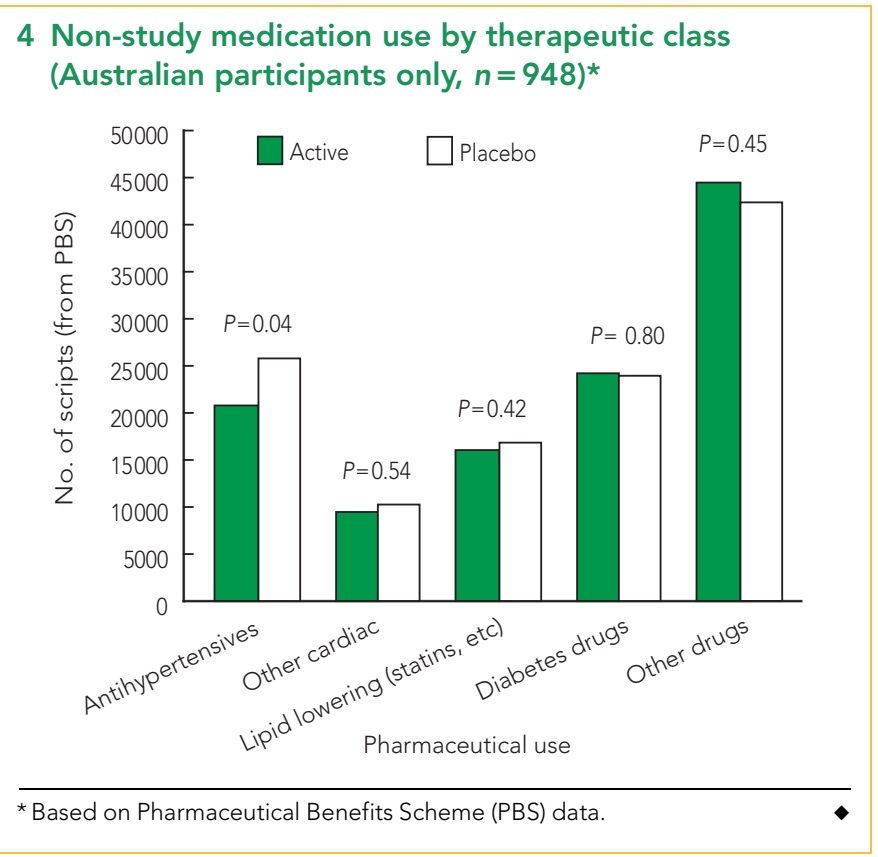

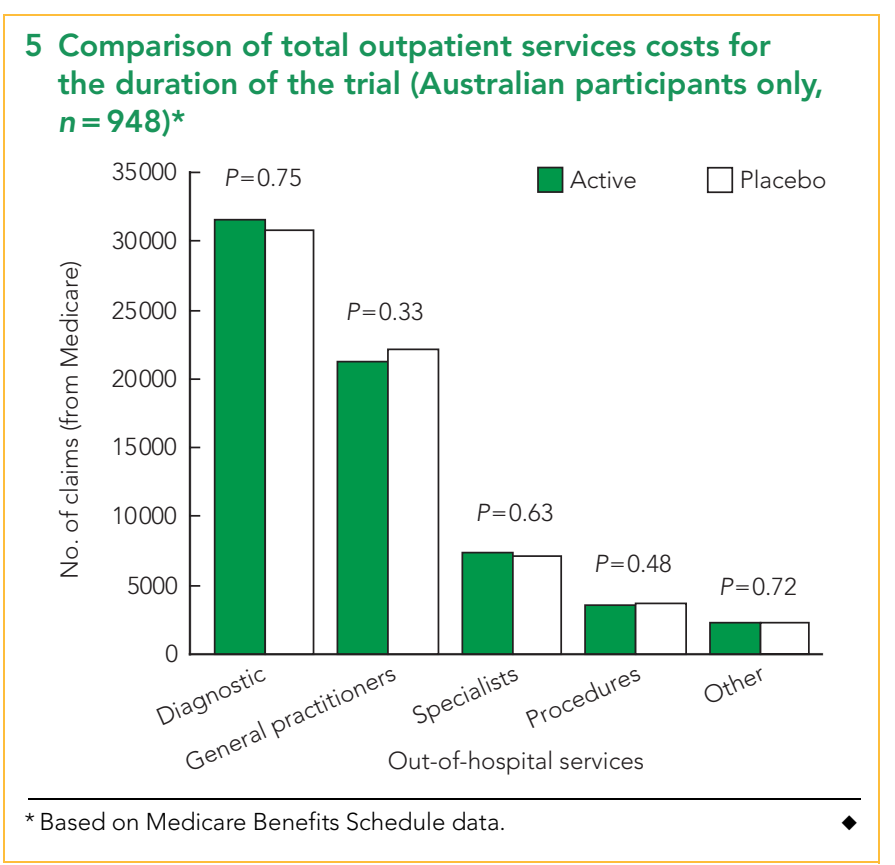

difference per patient treated was $\mathrm{A} \$ 555$. Hence the cost per death prevented was around A\$49 200.

Predicted survival times for both treatment groups were substantially shorter than those for the general population of the same age. Within the period of follow-up, participants allocated to the perindopril-indapamide intervention lived a mean of 4.17 years (95\% CI, 4.15-4.18 years) compared with 4.14 years (95\% CI, 4.12-4.16 years) for the placebo group - an incremental gain in life expectancy of 0.03 years $(95 \% \mathrm{CI}, 0.00$ 0.05 years) (Box 6). Based on the observed within-trial treatment effects of perindoprilindapamide, the modelled mean life expectancy from the date of randomisation was 15.0 years ( $95 \% \mathrm{CI}, 13.8-16.4$ years) in the intervention group and 14.9 years $(95 \% \mathrm{CI}$, $13.7-16.4$ years) in the placebo group, a difference of 0.09 years ( $95 \%$ CI, 0.06-0.12

6 Life expectancy and life-years gained, from the within-trial effect of treatment with fixed combination of perindopril and indapamide

\begin{tabular}{lccc} 
& \multicolumn{2}{c}{$\begin{array}{c}\text { Mean }(95 \% \mathrm{Cl}) \text { life expectancy (years) } \\
\text { per patient }\end{array}$} & \\
\cline { 2 - 3 } & \multicolumn{2}{c}{$\begin{array}{c}\text { Perindopril- } \\
\text { indapamide }\end{array}$} & $\begin{array}{c}\text { Mean life-year gain } \\
\text { per patient }(95 \% \mathrm{Cl})\end{array}$ \\
\hline Type of outcome & $4.17(4.15-4.18)$ & $4.14(4.12-4.16)$ & $0.03(0.00-0.05)$ \\
Within-trial & $14.97(13.77-16.44)$ & $14.88(13.69-16.35)$ & $0.09(0.06-0.12)$ \\
Total life expectancy & $12.28(11.48-13.23)$ & $12.22(11.42-13.17)$ & $0.06(0.04-0.07)$ \\
3\% discount per year & $10.88(10.27-11.60)$ & $10.84(10.23-11.56)$ & $0.05(0.03-0.06)$ \\
5\% discount per year & $8.36(8.04-8.72)$ & $8.34(8.01-8.70)$ & $0.02(0.02-0.03)$ \\
10\% discount per year & & &
\end{tabular}

ticipants, the incremental cost of the therapy was $\mathrm{A} \$ 1176$ (A\$1368-A $\$ 192$ [the offset by a reduction in hospital costs]). When costs and effects were discounted at $3 \%$, the incremental cost-effectiveness ratio was around $\mathrm{A} \$ 19800$ per life-year saved.

In the United Kingdom and United States, the price for 30 days' medication is $£ 14.49$ and US\$42, respectively. Assuming a similar proportional reduction in hospital and other costs, the UK and US costs per QALY would be about $£ 4085$ and US\$11842 per discounted life-year saved.

\section{DISCUSSION}

Our cost-effectiveness analysis of data from the ADVANCE trial found that the cost offset attributable to the intervention was roughly two-thirds of the total costs of perindoprilindapamide dispensed during the trial. The resultant cost-effectiveness of A $\$ 49200$ per premature death prevented is within a range generally considered acceptable and is comparable to that of many other interventions. There was no difference in quality of life between groups, but applying the average quality-of-life score results in a cost per QALY of around \$10600.

These findings are comparable to previous studies of BP reduction in patients with diabetes and patients at high risk of cardiovascular events. For example, in the UK Prospective Diabetes Study, the estimated cost per QALY was $£ 1049$ (in 1998 pounds). ${ }^{17}$ For participants with stable cor- 
onary disease in the EUROPA trial, the estimated cost per QALY of perindopril alone was $£ 9700 .^{18}$ Similar relative risk reductions were found across different age, sex, and lipid profile groupings. ${ }^{17,18}$ Absolute risk, and hence cost-effectiveness, is therefore largely dependent on individual predicted risk.

Although we could make some approximation of cost-effectiveness in the UK and US from the ADVANCE trial data, differences in health care systems would require additional analyses to extend this analysis to other countries in more detail. The absolute benefit is unlikely to be substantially influenced by country-specific factors and, given a similar price for perindopril-indapamide in other countries, the cost offsets are unlikely to repay the medication costs. However, such country-specific variations are still likely to leave perindopril-indapamide in the cost-effective range.

If the monthly costs of medication were reduced to around $\$ 12$, then this intervention would reduce net costs as well as being clinically important. However, as it is, from the Australian payer's perspective, perindopril-indapamide is clearly cost-effective for patients with type 2 diabetes mellitus, independent of their BP level.

\section{ACKNOWLEDGEMENTS}

We thank Peter Walker and 3M Australia for granting us use of their software for our analyses, the ADVANCE centres, and the ADVANCE trial patients. The ADVANCE trial is funded by grants from the National Health and Medical Research Council of Australia and Institut de Recherches Internationales Servier. Members of the ADVANCE Collaborative Group are fully listed in The Lancet. ${ }^{9}$ Neil Poulter received funding from the UK National Institute for Health Research Biomedical Research Centre funding scheme. The George Institute provided institutional grants for work on the substudy by Paul Glasziou, Philip Clarke, Janet Alexander, Mohana Rajmokan, and Elaine Beller. John Chalmers and Philip Clarke received institutional research grants from Servier.

\section{COMPETING INTERESTS}

Paul Glasziou was reimbursed by the George Institute for travel to management committee meetings. John Chalmers is paid as a Board Member of the Servier International Diabetes Advisory Board. John Chalmers, Anushka Patel, Neil Poulter and Mark Woodward have received honoraria from Servier for speaking at scientific meetings. Mark Woodward has been paid by Roche as a member of the dal-PLAQUE steering committee and as a consultant by Servier, AstraZeneca and GlaxoSmithKline.

\section{AUTHOR DETAILS}

Paul P Glasziou, MB BS, PhD, Director ${ }^{1}$

Philip M Clarke, MEc, PhD, Associate Professor, Department of Public Health ${ }^{2}$

Jan Alexander, Data Manager ${ }^{3}$

Mohana Rajmokan, MSc, Statistician ${ }^{4}$

Elaine Beller, BSc, MAppStat, Director ${ }^{3}$

Mark Woodward, PhD, Professor of

Biostatistics ${ }^{2,5}$

John Chalmers, MD, PhD, FRACP, Emeritus

Professor of Medicine, ${ }^{2}$ and Senior Director ${ }^{5}$

Neil Poulter, MSc, FRCP, Professor of

Preventive Cardiovascular Medicine ${ }^{6}$

Anushka A Patel, FRACP, PhD, Director,

Cardiovascular Division ${ }^{5}$

1 Clinical Epidemiology and Biostatistics, Bond University, Gold Coast, QLD.

2 University of Sydney, Sydney, NSW.

3 Queensland Clinical Trials Centre, University of Queensland, Brisbane, QLD.

4 Centre for Healthcare Related Infection Surveillance and Prevention, Queensland Health, Brisbane, QLD.

5 The George Institute for International Health, Sydney, NSW.

6 Imperial College London, London, UK.

Correspondence: pglaszio@bond.edu.au

\section{REFERENCES}

1 Wild S, Roglic G, Green A, et al. Global prevalence of diabetes: estimates for the year 2000 and projections for 2030. Diabetes Care 2004; 27: 1047-1053.

2 Turnbull F, Neal B, Pfeffer $M$, et al. Blood pressure-dependent and independent effects of agents that inhibit the renin-angiotensin system. J Hypertens 2007; 25: 951-958.

3 Gu D, Reynolds K, Wu X, et al; InterASIA Collaborative Group. Prevalence, awareness, treatment, and control of hypertension in China. Hypertension 2002; 40: 920-927.

4 Ong KL, Cheung BM, Man YB, et al. Prevalence, awareness, treatment, and control of hypertension among United States adults 19992004. Hypertension 2007; 49: 69-75.

5 Patel R, Lawlor DA, Whincup $P$, et al. The detection, treatment and control of high blood pressure in older British adults: cross-sectional findings from the British Women's Heart and Health Study and the British Regional Heart Study. J Hum Hypertens 2006; 20: 733-741.

6 Baker S, Priest P, Jackson R. Using thresholds based on risk of cardiovascular disease to target treatment for hypertension: modelling events averted and number treated. BMJ 2000; 320: 680-685

7 Asia Pacific Cohort Studies Collaboration, Kengne AP, Patel A, Barzi F, et al. Systolic blood pressure, diabetes and the risk of cardiovascular diseases in the Asia-Pacific region. $J$ Hypertens 2007; 25: 1205-1213.

8 Patel A, MacMahon S, Chalmers J, et al: ADVANCE Collaborative Group. Effects of a fixed combination of perindopril and indapamide on macrovascular and microvascular outcomes in patients with type 2 diabetes mellitus (the ADVANCE trial): a randomised controlled trial. Lancet 2007; 370: 829-840.

9 Rationale and design of the ADVANCE study: a randomised trial of blood pressure lowering and intensive glucose control in high-risk individuals with type 2 diabetes mellitus. Action in diabetes and vascular disease: preterax and diamicron modified-release controlled evaluation. J Hypertens Suppl 2001; 19: S21-28.

10 Study rationale and design of ADVANCE: action in diabetes and vascular disease preterax and diamicron MR controlled evaluation. Diabetologia 2001; 44: 1118-1120.

11 Gudex C, Kind P. The QALY toolkit. (Discussion paper 38.) York: University of York, Centre for Health Economics, 1988.

12 Ware JE, Kosinski M, Dewey J. How to score version two of the SF-36 health survey. Lincoln, RI: QualityMetric Incorporated, 2000.

13 Glasziou P, Alexander J, Beller E, Clarke P. Which health-related quality of life score? A comparison of alternative utility measures in patients with type 2 diabetes in the ADVANCE trial. Health Qual Life Outcomes 2007; 5: 21.

14 Department of Health and Ageing. National Hospital Cost Data Collection (NHCDC) reports. 2007. Round 11 cost weights, peer group report and hospital reference manual for 2006-07. Canberra: DoHA, 2008. http:// www.health.gov.au/internet/main/publishing.nsf/Content/health-casemix-data-collections-NHCDCReports (accessed Dec 2007).

15 Dolan P, Roberts J. Modelling valuations for Eq$5 d$ health states: an alternative model using differences in valuations. Med Care 2002; 40: 442-446.

16 Clarke PM, Glasziou P, Patel A, et al. Event rates, hospital utilization and costs associated with major complications of diabetes: multicountry comparative analysis. PLoS Med 2010; 7: e1000236. doi: 10.1371/journal.pmed.100 0236.

17 Raikou M, Gray A, Briggs A, et al; UK Prospective Diabetes Study Group. Cost effectiveness analysis of improved blood pressure control in hypertensive patients with type 2 diabetes: UKPDS 40. BMJ 1998; 317: 720-726.

18 Briggs A, Mihaylova B, Sculpher M, et al; EUROPA Trial investigators. Cost effectiveness of perindopril in reducing cardiovascular events in patients with stable coronary artery disease using data from the EUROPA study. Heart 2007; 93: 1081-1086.

(Received 26 Jan 2010, accepted 6 Jun 2010) $\square$ 